\title{
A New Approach for Isomorphism Identification among Compound Kinematic Chains and Mechanisms
}

\author{
Ashok Dargar \\ Department of Mechanical Engineering, School of Engineering, Sir Padampat Singhania University \\ NH-76, Udaipur- 313601, India. \\ *Corresponding author: dargarashok@rediffmail.com
}

Copyright $@ 2013$ Horizon Research Publishing All rights reserved.

\begin{abstract}
Structure synthesis of mechanisms is a pivotal issue in the field of mechanical innovation and mechanical conceptual design. The present work deals with problem of detection of isomorphism which is frequently encountered in structural synthesis of kinematic chains. A new approach based on a combination of eigenvalues and eigenvectors which are further associated with the modified adjacency matrix is proposed. The proposed method not only effective but also more efficient than that are based on the adjacency matrices and capable of detecting isomorphism in all types of compound $\mathrm{KC}$, i.e. chains of single or multi degree of freedom with simple or multiple joints. This study will help the designer to select the best $\mathrm{KC}$ and mechanisms to perform the specified task at conceptual stage of design. Some examples are provided to demonstrate the effectiveness of this method.
\end{abstract}

Keywords Kinematic Chain (KC), Distinct Mechanisms, Eigenvalues, Eigenvectors

\section{Introduction}

One of the most creative and important stages in mechanical design is the topological structure synthesis of mechanisms. The structure synthesis is the original innovation of mechanical systems, which not only can optimize existing mechanisms, but also invent new ones. Structure synthesis of kinematic chains usually generates chains in batch, which must be followed by isomorphism identification to eliminate duplicate kinematic chains. Isomorphism identification between kinematic chains is one of the most fascinating and challenging problems in mechanism and has been studied for about 50 years. Many attempts have been made in literature to develop reliable and computationally efficient tests for isomorphism. Uicker and Raicu [1] investigated the properties of the characteristic polynomial of the adjacent matrix of a kinematic chain. Murthyunjaya and Raghavan [2] applied Bocher's formula for the determination of the characteristic coefficients and presented a counter example for the uniqueness of the characteristic polynomial. Yan and Hall [3, 4] presented rules and theorems by which characteristic polynomial of kinematic chain and its coefficients are determined. Yan and Hwang [5] defined the linkage path code of a kinematic chain. Mruthyunjaya and Balasubramanian [6] worked on characteristic polynomial of a vertex-vertex degree matrix; they brought light on counter examples. Ambekar and Agrawal [7] proposed max code and min code methods for the detection of isomorphism. Shin and Krishnamurthy [8] presented the standard code theory for the detection of isomorphism. Shende and Rao [9] proposed a method based on summation polynomials. Rao and Varadaraju [10] defined link Hamming string as an index for testing isomorphism. Yadav et. al [11] presented a sequential three-step test for isomorphism. Chang et.al [12] presented a new method based on eigen values and eigen vectors of adjacent matrices of chains. Cubillo and Wan [13] stated the necessary and sufficiency conditions of eigenvalues and eigenvectors to identity isomorphic chains. Sunkari and Schmidt [14] worked on the reliability and efficiency of the existing spectral methods. Ding and Huang $[15,16]$ addresses the problem of isomorphism identification by finding a unique representation of graphs. Recently Dargar et al. presented methods based on higher adjacency of links of kinematic chain [17-21]. However with regard to these methods, either there is a lack of uniqueness or they take too much time to determine the isomorphism among $\mathrm{KCs}$ and inversions of a $\mathrm{KC}$. Hence, there is a need to develop a computationally efficient method.

Critical study of kinematic chain and mechanism structure has revealed that the performance of the joints is affected by the degree of links and type of joints. In this paper considering these facts a new matrix called as modified adjacency matrix is proposed and a new approach based on a combination of eigenvalue and eigenvector, as opposed to relevant methods in the literature which only make use of the eigenvalue is presented to detect isomorphism among kinematic chains and to determine the distinct mechanisms of a $\mathrm{KC}$. 


\section{Definitions of Terminology}

Following definitions are to be understood clearly before applying this method. Various definitions with their abbreviations are given below.

(i) Degree of link: A numerical value for the link, based on its connectivity to other links therefore quaternary link has degree equal to four and ternary link has degree equal to three.

(ii) Joint value: It is the ratio of summation of degree of all the links connected at the joint to the number of links connected at the joint (type of joint). it is denoted by $\mathrm{J}_{\mathrm{v}}$ and computed by Eq.(1).

$$
\mathrm{J}_{\mathrm{v}}=\frac{\text { DDegree of all the connected links }}{\text { number of links connected at the joint }}
$$

(iii) Link value: For a link it is defined as the sum of joint values of all the joints of that link. For example the Link Values of the link 1 is $(2.5+2.5+2.5=7.5)$ and for link 5 it is $(3+3+2.5=8.5)$ of the chain of Fig. 1 (a).

(iv) Modified adjacency matrix: For an n-link $\mathrm{KC}$, it is defined as an $\mathrm{n} \times \mathrm{n}$ square matrix whose any $\mathrm{i}^{\text {th }}$ and $\mathrm{j}^{\text {th }}$ element $\mathrm{M}_{\mathrm{ij}}$ is defined as

$$
[\mathrm{MAM}]=\left\{\mathrm{M}_{\mathrm{ij}}\right\} \mathrm{n} \times \mathrm{n},
$$

Where

$\mathrm{M}_{\mathrm{ij}\{}=$ Sum of the link values of adjacent link $\mathrm{i}$ and $\mathrm{j}$

$=0$ otherwise $\}$

$$
[\mathrm{MAM}]=\left[\begin{array}{ccccc}
0 & \mathrm{M} 12 & \mathrm{M} 13 & \mathrm{M} 14 & \mathrm{M} 1 n \\
\text { M21 } & 0 & \mathrm{M} 23 & \mathrm{M} 24 & \text { M2n } \\
\text { M31 } & \mathrm{M} 32 & 0 & \text { M34 } & \text { M3n } \\
- & - & - & 0 & - \\
\mathrm{Mn} 1 & \mathrm{Mn} 2 & \mathrm{Mn} 3 & \mathrm{Mn} 4 & 0
\end{array}\right]
$$

\section{Basic Theory}

The characteristic polynomial [1] is generally derived from $(0,1)$ adjacency matrix. The roots of $n^{\text {th }}$ order characteristic polynomial are the set of n-Eigen values called as Eigen spectrum. Many researchers have reported co-spectral graphs (the non-isomorphic graphs having same Eigen spectrum derived from $(0,1)$ adjacency matrix). The proposed Modified Adjacency Matrix has additional information about the link values of adjacent links of a $\mathrm{KC}$. Therefore, it is expected that the characteristic polynomial and its coefficients will be unique to clearly identify the $\mathrm{KC}$ and even $\mathrm{KC}$ with co-spectral graphs.

\subsection{Test for Isomorphism of Kinematic Chains}

Theorem [22] : Two similar matrices, A and B, have the same Eigenvalues. Furthermore, if the similarity transformation from $\mathrm{A}$ to $\mathrm{B}$ is $\mathrm{B}=\mathrm{P}^{-1} \mathrm{AP}$, then the Eigenvectors, $\mathrm{X}$ and $\mathrm{Y}$, of $\mathrm{A}$ and $\mathrm{B}$ respectively are related by the equation

$$
\mathrm{Y}=\mathrm{P}^{-1} \mathrm{X}
$$

Let the two $\mathrm{KC}$ are represented by the two similar matrices $\mathrm{A}$ and $\mathrm{B}$. The Eigenvalues, $\lambda$ and the Eigenvectors, $\mathrm{X}$, of $\mathrm{A}$ satisfy the relationship $\mathrm{AX}=\lambda \mathrm{X}$.

Hence, $\mathrm{P}^{-1} \mathrm{AX}=\lambda \mathrm{P}^{-1} \mathrm{X}$

Secondly, using the fact that $\mathrm{PP}^{-1}=\mathrm{I}$, we have $\mathrm{P}^{-1} \mathrm{AP} \mathrm{P}^{-1} \mathrm{X}=$ $\lambda \mathrm{P}^{-1} \mathrm{X}$, Which may be written $\left(\mathrm{P}^{-1} \mathrm{AP}\right)\left(\mathrm{P}^{-1} \mathrm{X}\right)=\left(\lambda \mathrm{P}^{-1} \mathrm{X}\right)$ or $\mathrm{BY}=\lambda \mathrm{Y}$, Where $\mathrm{B}=\mathrm{P}^{-1} \mathrm{AP}$ and $\mathrm{Y}=\mathrm{P}^{-1} \mathrm{X}$

This shows that the Eigenvalues of $A$ are also the Eigenvalues of $\mathrm{B}$ and that the Eigenvectors of $\mathrm{B}$ are of the form $\mathrm{P}^{-1} \mathrm{X}$. It means that two kinematic chains are isomorphic if and only if Eigenvectors of their Modified Adjacency Matrices have the relation of $\mathrm{Y}=\mathrm{P}^{-1} \mathrm{X}$. So Eigenvalues and Eigenvectors of two isomorphic kinematic chains are equal in correspondence.

\subsection{Procedure to Identify Isomorphic Kinematic Chains}

(i) Write down the modified adjacency matrices of kinematic chains.

(ii) Compute the eigenvalues and eigenvectors of modified adjacency matrices.

(iii) Compare the eigenvalues of modified adjacency matrices by visual inspection. If the eigenvalues are correspondingly equal then go to next step. Otherwise the chains are non-isomorphic.

(iv) Compare the eigenvectors; if the eigenvectors corresponding to single eigenvalue of modified adjacency matrices are correspondingly equal or prorata, then the chains are isomorphic otherwise, the chains are non-isomorphic. The procedure is illustrated more clearly by the following examples.

\subsection{Procedure to Identify Distinct Mechanisms of a Given Kinematic Chain}

(i) Write down the modified adjacency matrix of kinematic chain.

(ii) Compute the eigenvalues and eigenvectors of modified adjacency matrix.

(iii)Compare the eigenvectors; row-wise similarity of eigenvectors of corresponding links (without considering its sign) gives number of equivalent links and row-wise variation of eigenvectors of corresponding links (without considering its sign) leads to number of distinct mechanism of given chain. 


\section{Application to Kinematic Chains}

Example 1: The first example concerns two KCs with 12 links, 16 joints, single-degree of freedom as shown in Figs.1 (a) and 1 (b). The task is to examine whether these two $\mathrm{KC}$ are isomorphic.

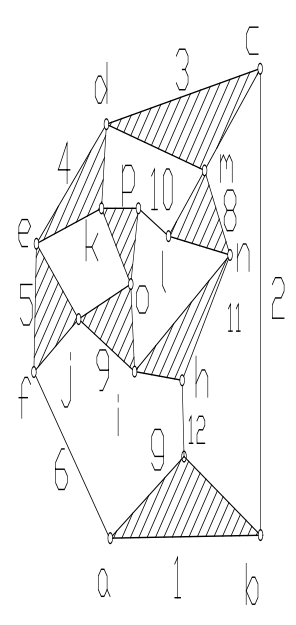

(a)

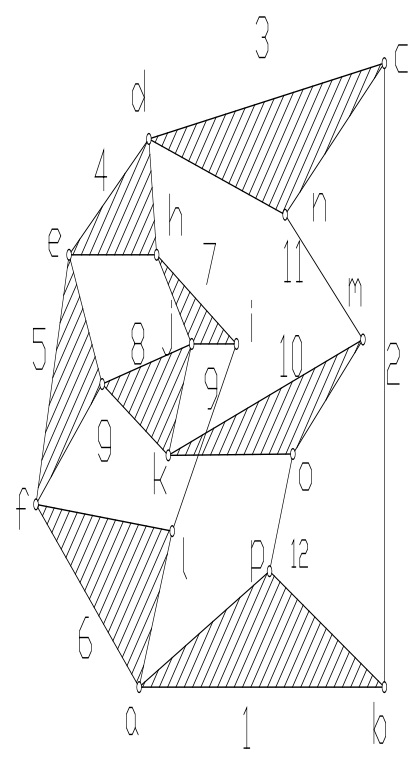

(b)

Figure 1. Twelve-links single degree of freedom kinematic chains

For chain of Fig. 1(a)

Link values of the different Links are; $\mathrm{L}_{1}=7.5, \mathrm{~L}_{2}=5, \mathrm{~L}_{3}=8.5, \mathrm{~L}_{4}=9, \mathrm{~L}_{5}=8.5, \mathrm{~L}_{6}=5, \mathrm{~L}_{7}=8.5, \mathrm{~L}_{8}=8.5, \mathrm{~L}_{9}=9, \mathrm{~L}_{10}=5, \mathrm{~L}_{11}=8.5$, $\mathrm{L}_{12}=5$.

Modified adjacency matrix:

$$
\left[\begin{array}{cccccccccccc}
0 & 12.5 & 0 & 0 & 0 & 12.5 & 0 & 0 & 0 & 0 & 0 & 12.5 \\
12.5 & 0 & 13.5 & 0 & 0 & 0 & 0 & 0 & 0 & 0 & 0 & 0 \\
0 & 13.5 & 0 & 17.5 & 0 & 0 & 0 & 17 & 0 & 0 & 0 & 0 \\
0 & 0 & 17.5 & 0 & 17.5 & 0 & 17.5 & 0 & 0 & 0 & 0 & 0 \\
0 & 0 & 0 & 17.5 & 0 & 13.5 & 0 & 0 & 17.5 & 0 & 0 & 0 \\
12.5 & 0 & 0 & 0 & 13.5 & 0 & 0 & 0 & 0 & 0 & 0 & 0 \\
0 & 0 & 0 & 17.5 & 0 & 0 & 0 & 0 & 17.5 & 13.5 & 0 & 0 \\
0 & 0 & 17 & 0 & 0 & 0 & 0 & 0 & 0 & 13.5 & 17 & 0 \\
0 & 0 & 0 & 0 & 17.5 & 0 & 17.5 & 0 & 0 & 0 & 17.5 & 0 \\
0 & 0 & 0 & 0 & 0 & 0 & 13.5 & 13.5 & 0 & 0 & 0 & 0 \\
0 & 0 & 0 & 0 & 0 & 0 & 0 & 17 & 17.5 & 0 & 0 & 13.5 \\
12.5 & 0 & 0 & 0 & 0 & 0 & 0 & 0 & 0 & 0 & 13.5 & 0
\end{array}\right]
$$

For chain of Fig. 1(b)

Link values of the different Links are; $\mathrm{L}_{1}=8, \mathrm{~L}_{2}=5, \mathrm{~L}_{3}=8, \mathrm{~L}_{4}=9, \mathrm{~L}_{5}=9, \mathrm{~L}_{6}=8.5, \mathrm{~L}_{7}=8.5, \mathrm{~L}_{8}=9, \mathrm{~L}_{9}=5, \mathrm{~L}_{10}=8, \mathrm{~L}_{11}=5, \mathrm{~L}_{12}=$ 5

Modified adjacency matrix:

$$
\left[\begin{array}{cccccccccccc}
\mathbf{0} & 13 & \mathbf{0} & \mathbf{0} & \mathbf{0} & \mathbf{1 6 . 5} & \mathbf{0} & \mathbf{0} & \mathbf{0} & \mathbf{0} & \mathbf{0} & \mathbf{1 3} \\
\mathbf{1 3} & \mathbf{0} & \mathbf{1 3} & \mathbf{0} & \mathbf{0} & \mathbf{0} & \mathbf{0} & \mathbf{0} & \mathbf{0} & \mathbf{0} & \mathbf{0} & \mathbf{0} \\
\mathbf{0} & \mathbf{1 3} & \mathbf{0} & \mathbf{1 7} & \mathbf{0} & \mathbf{0} & \mathbf{0} & \mathbf{0} & \mathbf{0} & \mathbf{0} & \mathbf{1 3} & \mathbf{0} \\
\mathbf{0} & \mathbf{0} & \mathbf{1 7} & \mathbf{0} & \mathbf{1 8} & \mathbf{0} & \mathbf{1 8} & \mathbf{0} & \mathbf{0} & \mathbf{0} & \mathbf{0} & \mathbf{0} \\
\mathbf{0} & \mathbf{0} & \mathbf{0} & \mathbf{1 8} & \mathbf{0} & \mathbf{1 7 . 5} & \mathbf{0} & \mathbf{1 8} & \mathbf{0} & \mathbf{0} & \mathbf{0} & \mathbf{0} \\
\mathbf{1 6 . 5} & \mathbf{0} & \mathbf{0} & \mathbf{0} & \mathbf{1 7 . 5} & \mathbf{0} & \mathbf{0} & \mathbf{0} & \mathbf{1 3 . 5} & \mathbf{0} & \mathbf{0} & \mathbf{0} \\
\mathbf{0} & \mathbf{0} & \mathbf{0} & \mathbf{1 8} & \mathbf{0} & \mathbf{0} & \mathbf{0} & \mathbf{1 8} & \mathbf{1 4} & \mathbf{0} & \mathbf{0} & \mathbf{0} \\
\mathbf{0} & \mathbf{0} & \mathbf{0} & \mathbf{0} & \mathbf{1 8} & \mathbf{0} & \mathbf{1 8} & \mathbf{0} & \mathbf{0} & \mathbf{1 7} & \mathbf{0} & \mathbf{0} \\
\mathbf{0} & \mathbf{0} & \mathbf{0} & \mathbf{0} & \mathbf{0} & \mathbf{1 3 . 5} & \mathbf{1 4} & \mathbf{0} & \mathbf{0} & \mathbf{0} & \mathbf{0} & \mathbf{0} \\
\mathbf{0} & \mathbf{0} & \mathbf{0} & \mathbf{0} & \mathbf{0} & \mathbf{0} & \mathbf{0} & \mathbf{1 7} & \mathbf{0} & \mathbf{0} & \mathbf{1 3} & \mathbf{1 3} \\
\mathbf{0} & \mathbf{0} & \mathbf{1 3} & \mathbf{0} & \mathbf{0} & \mathbf{0} & \mathbf{0} & \mathbf{0} & \mathbf{0} & \mathbf{1 3} & \mathbf{0} & \mathbf{0} \\
13 & \mathbf{0} & \mathbf{0} & \mathbf{0} & \mathbf{0} & \mathbf{0} & \mathbf{0} & \mathbf{0} & \mathbf{0} & \mathbf{0} & \mathbf{1 3} & \mathbf{0}
\end{array}\right]
$$


Table 1. Eigenvalues and Eigenvectors of chain 1 (a)

\begin{tabular}{|c|c|c|c|c|c|c|c|c|c|c|c|}
\hline-43.231 & 29.767 & -22.102 & -20.743 & -13.090 & -0.0000 & 5.4703 & 8.9481 & 22.102 & 22.399 & 25.147 & 44.867 \\
\hline 0.1229 & $\begin{array}{l}-0.1650 \\
\end{array}$ & -0.0000 & 0.6471 & -0.1336 & -0.0000 & 0.0414 & -0.3547 & 0.0000 & -0.5656 & -0.2493 & -0.1074 \\
\hline-0.1317 & 0.2076 & 0.3054 & -0.3048 & 0.2793 & 0.5599 & 0.2953 & -0.0989 & 0.3054 & -0.2690 & -0.22900 & -0.1232 \\
\hline 0.3079 & $\cdot 0.3049$ & $-0,5000$ & $\cdot 0.1307$ & $\cdot 0.1472$ & 0.0000 & 0.0814 & 0.2629 & 0.5000 & 0.0773 & $-0,3094$ & .0 .3099 \\
\hline-0.4370 & $\begin{array}{l}-0.1631 \\
\end{array}$ & 0.3959 & 0.1114 & .0 .0366 & -0.4319 & 0.1786 & 0.0961 & 0.3959 & 0.0136 & 0.2406 & -0.4117 \\
\hline 0.4043 & 0.2019 & -0.0000 & 0.1140 & 0.5297 & -0.0000 & -0.2703 & 0.2913 & 0.0000 & -0.2653 & 0.3770 & -0.3630 \\
\hline$=0.1618$ & -0.0223 & 0.0000 & $\begin{array}{r}-0.4641 \\
\end{array}$ & .0 .4188 & .0 .0000 & .0 .5725 & 0.0561 & 0.0000 & .0 .4755 & 0.0785 & -0.1391 \\
\hline 0.3673 & 0.3805 & 0.0000 & -0.1154 & -0.3552 & 0.0000 & 0.2447 & -0.5050 & -0.0000 & 0.2054 & 0.2781 & .0 .3826 \\
\hline-0.2286 & 0.5370 & $-0,0000$ & 0.2869 & -0.0708 & $-0,0000$ & -0.3922 & 0.1180 & $-0,0000$ & 0.3015 & -0.4750 & -0.2963 \\
\hline-0.4370 & -0.1631 & -0.3959 & 0.1114 & -0.0366 & 0.4319 & 0.1786 & 0.0961 & -0.3959 & 0.0136 & 0.2406 & -0.4117 \\
\hline$=0,0433$ & -0.4161 & 0.0000 & -0.1116 & 0.4393 & $=0,0000$ & $\cdot 0.3638$ & $.0,5839$ & $=0,0000$ & 0.3055 & 0.1057 & 0.2043 \\
\hline 0.3079 & -0.3049 & 0.5000 & -0.1307 & -0.1472 & $\cdot 0.0000$ & 0.0814 & 0.2629 & -0.5000 & 0.0773 & $\begin{array}{ll}3 & -0.3094\end{array}$ & $4 \quad-0.3099$ \\
\hline-0.1317 & 0.2076 & -0.3054 & -0.3048 & 0.2793 & -0.5599 & 0.2953 & .0 .0989 & -0.3054 & -0.2690 & -0.2900 & $\begin{array}{ll}0.0 .1232\end{array}$ \\
\hline
\end{tabular}

Table 2. Eigenvalues and Eigenvectors of chain 1 (b)

\begin{tabular}{|c|c|c|c|c|c|c|c|c|c|c|c|}
\hline-43.719 & -29.759 & -21.802 & -21.400 & 12.950 & $-0,0000$ & 53991 & 9.4886 & 21,400 & 22.905 & 25,003 & 45.435 \\
\hline-0.1574 & -0.4811 & 0.1446 & -0.0000 & 0.4877 & (1) & -0.2424 & 0.0595 & 0.0000 & -0.0949 & 0.6197 & 0.1722 \\
\hline 0.1220 & 0.2129 & -0.3116 & -0.3037 & -0.2711 & 0.5617 & -0.2950 & 0.0979 & 0.3037 & 0.1849 & 0.3543 & 0.1136 \\
\hline-0.2528 & -0.0064 & 0.3779 & - & -0.2176 & 0.0000 & 0.1199 & 0.0120 & 0.5000 & $0.4207 \quad 0$ & 0.0616 & 0.2247 \\
\hline 0.4420 & -0.1560 & 0.0982 & -0.3972 & - & (1) & - & -0.0934 & 0.3972 & t) & -0.2293 & 0.4154 \\
\hline 0.4539 & -0.1243 & -0.4029 & 0.0000 & $\begin{array}{l}-0.1685 \\
\end{array}$ & -0.0000 & 0.0627 & -0.5757. & -0.0000 & -0.2286 & -0.0635 & 0.4429 \\
\hline 0.2249 & 0.5322 & 0.2999 & -0.0000 & 0.0444 & -0.0000 & 0.3855 & -0.1201 & -0.0000 & -0.4231 & 0.3809 & 0.2952 \\
\hline -0.3807 & 0.3881 & -0.0730 & 0.0000 & 0.3459 & 0.0000 & -0.2293 & 0.5152 & -0.0000 & -0.0921 & -0.3131 & 0.3936 \\
\hline 0.4420 & -0.1560 & 0.0982 & 0.3972 & 0.0390 & 0.4295 & -0.1780 & -0.0934 & -0.3972 & 0.0603 & -0.2293 & 0.4154 \\
\hline 0.0525 & -0.4240 & -0.1388 & 0.0000 & -0.4203 & 0.0000 & 0.3693 & 0.5892 & -0.0000 & -0.3057 & $7 \quad 0.0303$ & $\begin{array}{lll}3 & 0.2090\end{array}$ \\
\hline -0.2528 & -0.0064 & 0.3779 & -0.5000 & -0.2176 & 0.0000 & 0.1199 & 0.0120 & -0.5000 & 0.4207 & 0.0616 & 0.2247 \\
\hline 0.1504 & 0.0056 & -0.4507 & 0.0000 & 0.4369 & -0.0000 & 0.5776 & 0.0329 & 0.0000 & 0.4776 & 0.0641 & 0.1286 \\
\hline 0.1220 & 0.2129 & 0.3116 & 0.3037 & -0.2711 & -0.5617 & -0.2950 & 0.0979 & -0.3037 & 0.1849 & 90.3543 & 30.1136 \\
\hline
\end{tabular}

Example 2: The second example concerns of two kinematic chains with 10 links, 13 joints, single freedom as shown in Figs. 2 (a) and 2 (b). The task is to examine whether these two chains are isomorphic.

For chain 2 (a)

Link values of the different Links are; $\mathrm{L}_{1}=12.5, \mathrm{~L}_{2}=5.5, \mathrm{~L}_{3}=$ $7.5, \mathrm{~L}_{4}=5, \mathrm{~L}_{5}=8, \mathrm{~L}_{6}=8.5, \mathrm{~L}_{7}=9, \mathrm{~L}_{8}=5, \mathrm{~L}_{9}=5.5, \mathrm{~L}_{10}=5.5$

For chain 2(b)
Link values of the different Links are; $\mathrm{L}_{1}=12.5, \mathrm{~L}_{2}=5.5, \mathrm{~L}_{3}=$ $7.5, \mathrm{~L}_{4}=5, \mathrm{~L}_{5}=5, \mathrm{~L}_{6}=5, \mathrm{~L}_{7}=9, \mathrm{~L}_{8}=8.5, \mathrm{~L}_{9}=5.5, \mathrm{~L}_{10}=5.5$

MATLAB ver.7.2 is used to evaluate the eigenvalues and the eigenvectors of modified adjacency matrices. This method reports that chain 1 (a) and (b) are non-isomorphic as the corresponding eigenvalues, listed in Table 1 and Table 2 are different for both the chains. The chains 2 (a) and 2 (b) are isomorphic as both eigenvalues and eigenvectors listed in Table 3 and Table 4, corresponding to single eigenvalue are correspondingly equal. Kong and Zhang [23] and Chang et al. (2002) also reported the same results, proving reliability of this method.

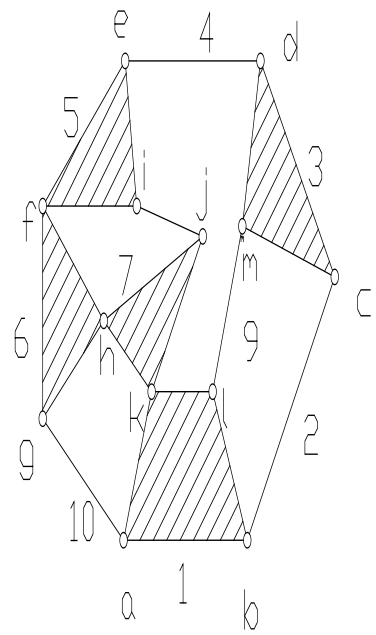

(a)

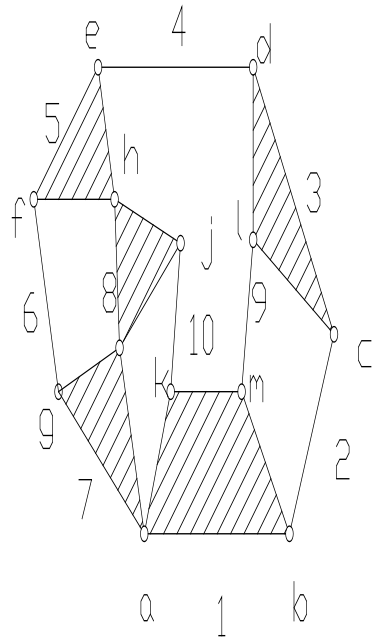

(b)
Figure 2. Ten Link Three degree of freedom kinematic chains

Table 3. Eigenvalues and Eigenvectors of chain 2 (a)

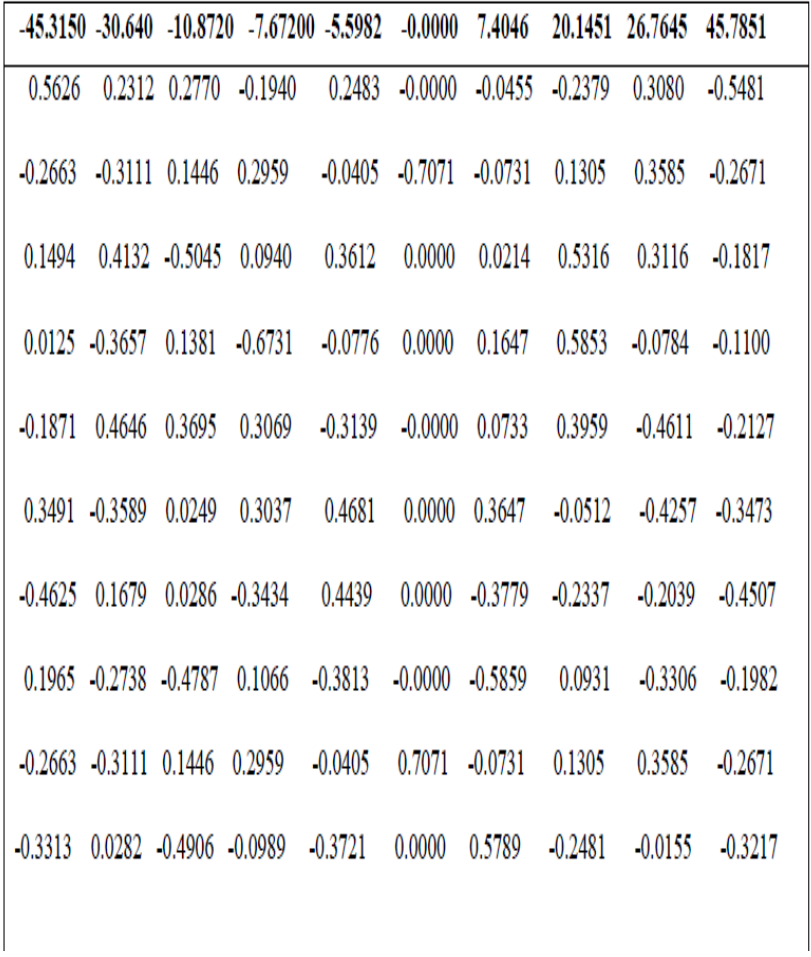


Table 4. Eigenvalues and Eigenvectors of chain 2 (b)

\begin{tabular}{|c|c|c|c|c|c|c|c|c|c|}
\hline-45.3150 & -30.640 & -10.8725 & -7.6728 & $-5,5982$ & -0.0000 & 7.4046 & 20.1451 & 26.7645 & 45.7851 \\
\hline 0.5626 & 0.2312 & 0.2770 & -0.1940 & 0.2483 & -0.0000 & -0.0455 & -0.2379 & 0.3080 & -0.5481 \\
\hline$\cdot 0.2663$ & -0.3111 & 0.1446 & 0.2959 & -0.0405 & -0.7071 & -0.0731 & 0.1305 & 0.3585 & -0.2671 \\
\hline 0.1494 & 0.4132 & -0.5045 & 0.0940 & 0.3612 & 0.0000 & 0.0214 & 0.5316 & 0.3116 & -0.1817 \\
\hline 0.0125 & -0.3657 & 0.1381 & -0.6731 & -0.0776 & 0.0000 & 0.1647 & 0.5853 & -0.0784 & -0.1100 \\
\hline -0.1871 & 0.4646 & 0.3695 & 0.3069 & -0.3139 & -0.0000 & 0.0733 & 0.3959 & -0.4611 & -0.2127 \\
\hline 0.3491 & -0.3589 & 0.0249 & 0.3037 & 0.4681 & 0.0000 & 0.3647 & -0.0512 & -0.4257 & -0.3473 \\
\hline$\cdot 0.4625$ & 0.1679 & 0.0286 & -0.3434 & 0.4439 & 0.0000 & -0.3779 & -0.2337 & -0.2039 & -0.4507 \\
\hline 0.1965 & -0.2738 & $\cdot 0.4787$ & 0.1066 & -0.3813 & -0.0000 & -0.5859 & 0.0931 & -0.3306 & -0.1982 \\
\hline$\cdot 0.2663$ & -0.3111 & 0.1446 & 0.2959 & -0.0405 & 0.7071 & -0.0731 & 0.1305 & 0.3585 & -0.2671 \\
\hline$\cdot 0.3313$ & 0.0282 & 0.04906 & -0.0989 & -0.3721 & 0.0000 & 0.5789 & -0.2481 & -0.0155 & -0.3217 \\
\hline
\end{tabular}

Example 3: This example concerns with 9-links, 11-joints, and two -degree of freedom kinematic chain shown in figure 3. The task is to determine number of possible distinct mechanisms of the chain.

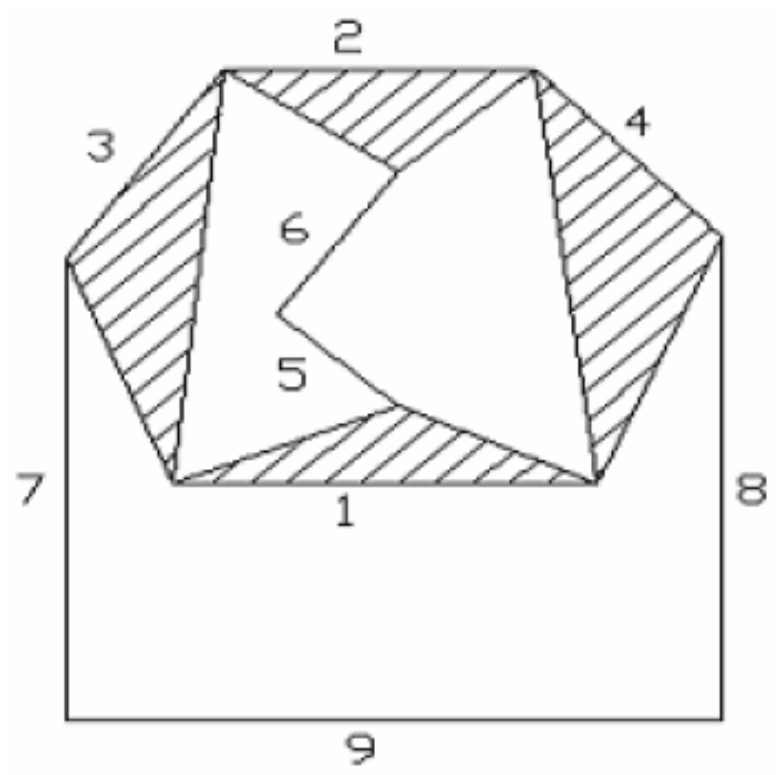

Figure 3. Nine Link Two degree of freedom kinematic chain

Identification of the distinct mechanisms: Observing the row wise similarity of Eigenvectors of corresponding links (without considering its sign) listed in Table 5, it is found that the links $(1,2),(3,4),(5,6)$ and $(7,8)$ are equivalent links and forms the four distinct mechanism. Link 9 has the distinct eigenvectors, forms the fifth distinct mechanism. Therefore, 5 distinct mechanisms are obtained from kinematic chain shown in figure 3.

Table 5. Eigenvalues and Eigenvectors of chain 3

\begin{tabular}{|c|c|c|c|c|c|c|c|c|}
\hline-38.246 & $6-18.256$ & $6-13.000$ & -11.750 & $\begin{array}{ll}06.333 \\
0\end{array}$ & 9.256 & $\begin{array}{ll}6 & 13.000\end{array}$ & 13.481 & 39.182 \\
\hline-0.4685 & 0.4101 & -0.0000 & 0.1864 & 40.1104 & 0.5760 & $\begin{array}{lll}0 & 0.0000\end{array}$ & -0.1288 & -0.4658 \\
\hline-0.4685 & -0.4101 & 0.0000 & 0.1864 & 0.1104 & -0.5760 & $\begin{array}{ll}0 & 0.0000\end{array}$ & -0.1288 & -0.4658 \\
\hline 0.4777 & -0.0000 & 0.5000 & -0.0198 & 0.2264 & 0.0000 & 0.5000 & 0.0918 & -0.4601 \\
\hline 0.4777 & 0.0000 & -0.5000 & -0.0198 & 0.2264 & 0.0000 & -0.5000 & 0.0918 & -0.4601 \\
\hline 0.1289 & -0.5760 & -0.0000 & -0.1168 & -0.5383 & 0.4101 & 0.0000 & -0.3738 & -0.2006 \\
\hline 0.1289 & 0.5760 & 0.0000 & -0.1168 & -0.5383 & -0.4101 & 0.0000 & -0.3738 & -0.2006 \\
\hline-0.1802 & 0.0000 & -0.5000 & -0.4696 & -0.1786 & -0.0000 & $\begin{array}{ll}0 & 0.5000\end{array}$ & 0.4322 & $2-0.1685$ \\
\hline-0.1802 & -0.0000 & 0.5000 & $=0.4696$ & -0.1786 & 0.0000 & -0.5000 & 0.4322 & $2-0.1685$ \\
\hline 0.0801 & 0.0000 & -0.0000 & 0.6793 & -0.4793 & 0.0000 & -0.0000 & 0.5450 & -0.0731 \\
\hline
\end{tabular}

\section{Results}

Using the proposed method the number of mechanisms derived from the family of 1-F; 6-link, 8-link and 2-F 9 link chains are 5, 71 and 254 respectively. These results in agreement those reported already in the literature. The distinct mechanisms derived from the family of 1-F; 10-link 1842 .

\section{Conclusion}

In this paper, a simple and compact approach has been introduced for identification of isomorphism between kinematic chains and among inversions of a kinematic chain. The proposed method is easy to compute, reliable and capable to identifying all distinct mechanisms derived from a given kinematic chain and also able to detect the isomorphism among the $\mathrm{KC}$ having simple joints and even the $\mathrm{KC}$ with co-spectral graphs. Authors strongly believe that this approach provide a new concept on which a new identification system can be based. Such a new identification system would be extremely selective and would eliminate the possibility of duplicate identification for structurally different mechanisms. The inherent relation between structural invariants and the mechanisms need further study.

\section{REFERENCES}


[1] Uicker, J. J., Raieu. A.: Method for the identification and Recognition of Equivalence of Kinematic chains. Mech. Mach. Theory, 22 (2), 125-130 (1975).

[2] Mruthyunjaya T. S., Raghavan M.R. : Structural analysis of kinematic chains and mechanisms based on matrix representation", ASME, J.Mech. Design, 101 (3), 488-494 (1979).

[3] Yan, H. S., Hall, A. S: Linkage characteristic polynomials assembly theorems uniqueness. J .Mech. Design, ASME Trans, 104, 11-20 (1982).

[4] Yan, H. S., Hall, A. S.: Linkage characteristic polynomials: definition, coefficients by inspection. J .Mech. Design, ASME Trans, 103, 578-584 (1981).

[5] Yan, H. S., Hwang, W. M.: Linkage Path code Mech. Mach. Theory, 19, 425-429 (1984).

[6] Mruthyunjaya T. S., Balasubramanian H. R.: In Quest of reliable and efficient computational test for detection of isomorphism in kinematic chains. Mech. Mach. Theory, 22, 131-139 (1987).

[7] Ambekar, A. G., Agarwal, V. P.: Canonical Numbering of Kinematic Chains, Mechanisms, Path generators and Function generators Using min Codes. Mech. Mach. Theory, 22, 453-461(1987).

[8] Shin, J. K., Krishna Murthy: On identification and Conical Numberings of Pin jointed Kinematic Chains. J. Mech. Design, ASME Trans, 116, 1994, 182-188 (1994).

[9] Shende, S., Rao, A. C.: Isomorphism in kinematic chains. Mech. Mach. Theory, 29, 1065-1070 (1994).

[10] Rao, A. C., Varda Raju, D.: Application of the Hamming number technique to detect isomorphism Among Kinematic Chains and inversions. Mech. Mach. Theory, 26 (1), 55-75 (1991).

[11] Yadav, J. N., Pratap C. R.: Computer aided detection of isomorphism among kinematic chains and mechanisms using the link- link multiplicity distance concept. Mech. Mach. Theory, 31, 873-877 (1996).

[12] Chang, Z. et al.: A new method to mechanism kinematic chain isomorphism Identification. Mech. Mach. Theory, 37, 411-417 (2002).
[13] Cubillo, J. P., Jinbao Wan.: Comments on mechanism kinematics chain isomorphism identification using adjacent matrices. Mech and Mach Theory 40, 131-139 (2004).

[14] Sunkari, R P., Schmidt C, Linda: Reliability and Efficiency of the Existing Spectral Methods for Isomorphism Detection. J. Mech. Des. 128 (6): 1246-1252 (2006).

[15] Ding, H., Haung, Z.: Isomorphism identification of graphs: Especially for the graphs of kinematic chains. Mech. Mach. Theory, 44 (1) 122-139 (2009).

[16] Ding, H., Haung, Z.: The Establishment of the Canonical Perimeter Topological Graph of Kinematic Chains and Isomorphism Identification. J. Mech. Des. 129 (9), 915-923 (2007).

[17] Dargar, A., Khan, R. A., and Hasan, A., Some New Codes for Isomorphism Identification among Kinematic Chains and their Inversions, International Journal of Mechanisms and Robotic Systems, 1 (1), 49-67, (2013).

[18] Dargar, A., Khan, R. A., and Hasan, A., A Method for Identification of Isomorphism and Structural Properties of Kinematic Chains, International Journal of Materials and Structural Integrity , 5 (4), 376-388, (2011).

[19] Dargar, A., Khan, R. A., and Hasan, A. Application of Link Adjacency Values to Detect Isomorphism among Kinematic Chains, International Journal of Mechanics and Materials in Design, 6, 157-162, (2010) .

[20] Dargar, A., Khan, R. A., and Hasan, A., Mobility Analysis of Kinematic Chains, Kathmandu University Journal of Science, Engineering and Technology, 6(1) 25-32, 2010.

[21] Dargar, A., Khan, R. A., and Hasan, A., Identification of Isomorphism among Kinematic Chains and Inversions using Link Adjacency Values, International Journal of Mechanical and Materials Engineering , 4 (3), 309-315, 2009.

[22] Hsiung, C. Y., Mao. G. Y. Linear Algebra. World Scientific Publishing company Pvt. Ltd.USA, (1998).

[23] Kong, F. G., Li, Q. and Zhang, W. J.: An artificial neural network approach to mechanism kinematic chain isomorphism identification. Mech. Mach. Theory, 34, 271-283(1999). 